\title{
Vectorization of an Applicative Language: Current Results and Future Directions
}

\author{
David C. Cann \\ Computing Research Group, L-306 \\ Lawrence Livermore National Laboratory. \\ P.O. Box 808, Livermore, CA 94550 \\ cann@lll-crg.llnl.gov
}

\begin{abstract}
The most important and challenging problem facing researchers today is the effective use of parallel processors. Few would deny that the biggest obstacle is the inadequacy of current software tools-an inadequacy born of imperative programming. As an alternative, applicative and functional programming languages provide a clearier and simpler parallel programming model. They hide architectural idiosyncrasies, guarantee determinancy, enforce software engineering principles, and in many ways simplify compilation. Regrettably these languages have acquired a reputation for inefficiency. In this report, we show that applicative programs do not require special hardware for efficient execution, and with little effort can automatically exploit concurrent and vector processo's.
\end{abstract}

\section{Tomorrow and Beyond}

One cannot dispute ForTran's dominance in scientific computing, but is ForTRAN an appropriate tool for programming parallel machines? Because of current software investments, one would argue that it must suffice. But this does not answer the question, nor does it address the growing software crisis. Take a moment to reflect on the number of scientific and commercial applications using miltiprocessors today. The number is discouragingly small. For example, all the multiprocessor Crays at Lawrence Livermore National Laboratory are programmed as disjoint uniprocessors. If two jobs can coexist in memory, they will execute at the sare time. Unfortunately, the memory intensive jobs choke off users and idle processors.

So why are these memory intensive jobs running sequentially? First, hand parallelization of large ForTRAN programs is arduous and intractable; the potential for error is huge, as is the cost. Second, automatic parallelizing compilers, regardless of their capabilities, cannot change inherently sequential algorithms into parallel algorithms. At Livermore, most of the production codes are 20 years old and have an average length of 200,000 lines. "Dusty" and "sequential" do not even begin to describe them. Hence, one might conjecture that the best solution is to redesign and rewrite the critical applications. However, even this carries too great a cost. A more important concern, therefore, is tomorrow's application.

We feel that the only way to improve the software crisis and effectively use parallel architectures is to transcend the imperative programming model. That is, use languages that assist the writting of parallel algorithms, ease automatic parallelization, and guarantee determinancy. Few (if any) of these languages are available commercially, however, and only a small number are available to researchers. Of these, only a few have shown good performance: consider $[3,4,9]$.

In this report, we contribute to the growing interest in new programming methodologies $[1,10,12$, 13]. Our intent is to further emphasize that languages that do not rely on persistent state and side effects can effectively and automatically exploit conventional concurrent-vector architectures and rival the performance of Fortran. To this end, we compare the performance of SISAL[14], an applicative language for parallel numerical computation, and FORTRAN using four scientific benchmarks and two production codes.

For completeness, the next section briefly examines the applicative programming paradigm and introduces SISAL. Then in the remaining sections we describe the potential inefficiencies of applicative computation, introduce the required optimizations, briefly discuss the automatic parallelization of SISAL, present our current results on the Alliant $\mathrm{FX} / 80$, and consider future directions.

Before we begin, let us ponder an important question: How should we program tomorrow's machines, machines that will undoubtedly support parallel pro- 
cessing? As we will show, the applicative programming model is worth serious consideration.

\section{Truth and Beauty}

In this section we summarize the aesthetics of applicative computation and present an overview of SISAL. To begin, we enumerate the desired characteristics of a parallel programming language [1]. First, the language must insulate the programmer from the target machine. Deriving and expressing a parallel algorithm once is hard enough; one should not have to reprogram the algorithm for each new machine. Second, parallelism must be implicit in the semantics of the language. The programmer should not be responsible for managing concurrency. Third, when the programmer desires determinancy, the language must guarantee it. Regardless of the conditions of execution, a program that realizes a determinate algorithm should yield the same results for the same inputs.

In general, imperative languages fail to meet these requirements; however, applicative and functional languages do fit the mold [13]. For illustration we will introdice SISAL and discuss a few of its attributes. A SISAL program is a collection of user defined functions, each of which applies one or more intrinsic functions (such as addition, multiplication, etc.) or user defined functions to inputs. Further, all these functions must obey call by value semantics, which precludes the aliases and side effects that complicate imperative computing. This in itself insulates the applicative programmer from the idiosyncrasies of hardware. For example, without call by reference semantics ${ }^{1}$, the concept of data replaces memory and the concept of creation replaces update. SISAL programs compute by creating new data objects-objects that can never change.

Because the definition of SISAL banishes side effect and aliasing problems, its operational semantics is implicitly parallel. Only data dependencies control the order of function invocation, and these deperidencies are explicit in SISAL text. A SiSAL program is nothing more than a high-level data dependence graph, or one might call it a high-level specification. For example, consider the following SISAL excerpt and its data dependence graph shown in Figure 1.

$$
T:=\operatorname{sqrt}(b * b-4 * a * c) \text {; }
$$$$
R:=(T-b) /(2 * a) \text {; }
$$

In the graph, the boxes represent functions and the edges represent the data paths. Note that $T$ and $R$

\footnotetext{
'SISAL does not support a pointer data type, hence does not als.w addresses to be passed by value, which would result in pass by reference semantics.
}

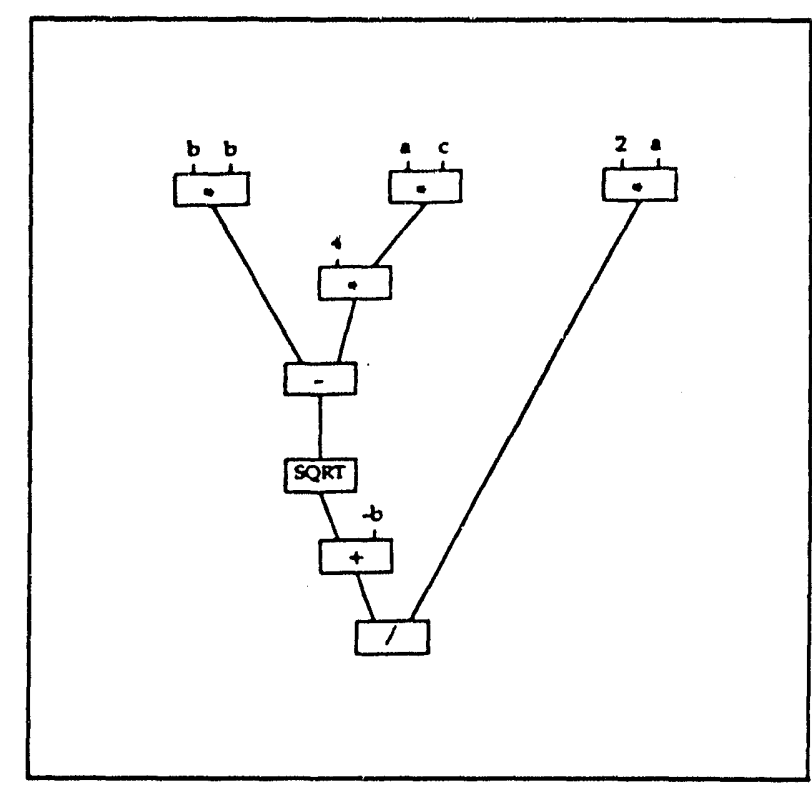

Figure 1. Example data dependence graph.

are nothing more than distinct edges in the graph; they are not memory locations. Further, note that the parallelism in the graph is explicit. In this paradigm, the top-left multiply can affect the top-right multiply only if an edge links the two operations. Such an edge does not exist in the example, so the two operations are truly independent and can execute concurrently. The availability of data defines the synchronization and coordination logic required to control their execution.

For the same reason that the operational semantics of SISAL is implicitly parallel. SISAL programs are determinate. That is, execution order is not random or a product of external events; it is a product of data dependencies and the availability of data.

So SISAL meets all the requirements of a parallel programming language. But truth and beauty aside, it might appear that applicative programming is too restrictive and onerous. Our experience has shown that applicative languages like SISAL are more than sufficient for expressing numerical computations. For example, Sisal supports all of the standard data types, including character, boolean, integer, real and double precision; as well as all of the standard aggregate types, including arrays, records and unions. Further, it defines a rich set of intrinsic functions, including compound operations for conditional (if), distributed (for), and iterative (for initial) definitions.

\section{Problems and Solutions}

In the previous section we saw that applicative languages, because of their semantics, meet all the requirements of a parallel programming language. However, 
Table 1. The benchmark suite (DP $=$ double precision, $\mathrm{R}=$ real, $\mathrm{CX}=$ complex, $\mathrm{C}=$ cycles $)$.

\begin{tabular}{|c||c|l|}
\hline Program & $\begin{array}{c}\text { Source } \\
\text { Lines }\end{array}$ & \multicolumn{1}{|c|}{$\begin{array}{c}\text { Execution } \\
\text { Comments }\end{array}$} \\
\hline \hline GAUSSJ & 114 & $100 \times 100, \mathrm{DP}$ \\
RICARD & 301 & $200 \mathrm{Cs}, 5 \times 1315, \mathrm{DP}$ \\
BMK11A & 1014 & 10Cs, $1 \times 2560, \mathrm{DP}$ \\
LOOPS & 1088 & $400 \mathrm{Cs}, \mathrm{DP}$ \\
SIMPLE & 1550 & /32Cs, 100x100, DP \\
WEATHER & 2718 & 10Cs, DP, R, CX \\
\hline
\end{tabular}

the data copying implied by call by value and side effect free semantics can render applicative computation infeasible, especially when building and manipulating large aggregates.

To illustrate the costs, let us consider the benchmark suite used in this report and its performance with and without the copy elimination optimizations discussed below. The suite itself is introduced in Table 1. We defer further description of the individual progroms until later. For now, it will suffice to say that all the programs are array and floating point intensive. Table 2 summarizes their performance with and without the optimizations. The times are in seconds and were gathered on an Alliant $\mathrm{FX} / 80$ using five processors (ACEs). We quickly draw two conclusions from the data: the cost of copying is truly exorbitant and the copy optimizations are dramatically effective.

So what are are these optimizations and when are they appropriate? For simplicity we will discuss only those functions that modify and build arrays. First, let us consider array modification:

$$
B:=S[3: K] \text {; }
$$

This expression changes the third component of array $S$ to $K$. Note that side effect free semantics implies that the update nust preserve $S$ and modify a copy. Fortunately, it is not always necessary to modify copies, and often the compiler can make this determination. For example, if the compiler can determine that the above update is the last use of $S$ in the program, then it can generate code to modify $S$ in-place. For some computations, the compiler can introduce artificial dependencies to force write operations to execute after read operations-thus further improving chances for copy elimination. In all. these optimizations fall under the heading of update-in-place analysis $[2,4,5,11,17]$.

Now let us consider array construction. We took the following for initial expression from SIMPLE:

$$
\begin{aligned}
B:= & \text { for initial } \\
r & :=\text { array oned []; }
\end{aligned}
$$

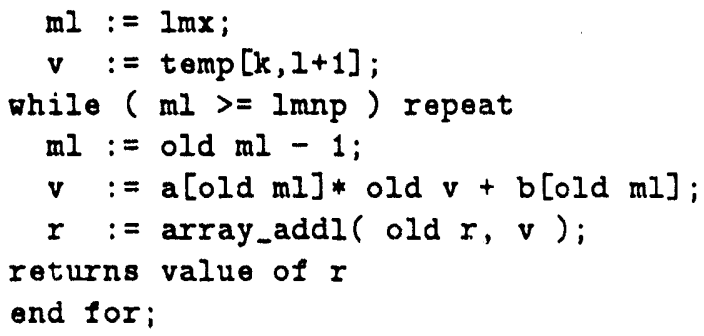

Each invocation of the "array_addl" operation builds a new array, one that is a single element larger than its predecessor. In total, $\mathbb{N + 2}$ arrays are built and $(\mathbb{N} * \mathbb{N}-\mathbb{N}) / 2$ values are copied (assuming $\mathbb{N}$ is $\operatorname{lm} x-\operatorname{lm} n \mathrm{p}$ ). For most computations, however, the intermediate arrays are superfluous. In the example, the compiler can generate code to precalculate the size of $B$ (which is $N+1$ ), preallocate its storage, and build its components in-place. Fittingly, we refer to this optimization as build-in-place analysis $[9,16]$.

In SISAL, there are other sources of intermediate array constructions that are subject to build-in-place analysis. For example, the following computation uses concatenation to add a zero to the array built in the previous example:

$$
\begin{aligned}
& W:=\operatorname{array}[1: 0.0] ; \\
& Z:=W \| B ;
\end{aligned}
$$

Semantically this computation must first build both B and $W$, and then copy both arrays to form $Z$. Unoptimized, this results in $(\mathbb{N} * \mathbb{N}+\mathbb{N}+2) / 2$ copy operations and the construction of $N+4$ arrays. But knowing the final size of the resulting array $(\mathrm{N}+2)$ the compiler can preallocate storage for $\mathbf{Z}$ and direct the array generator building $W$ and the for initial expression building $B$ to place their results into the storage. This eliminates all the intermediate arrays and the concatenation itself.

\section{Exploiting Applicative Paral- lelism}

We have already noted that parallelism is implicit in the operational semantics of an applicative language. As such, the automatic detection of implicit program parallelism is unnecessary. The applicative compilation system must only determine the amount of parallelism to exploit and decide how to map this parallelism to the target machine. For languages like SISAL and target machines like the Alliant, this determination is trivial. For example the most common source of parallelism in a SISAL program is the for expression, whose semantics precludes the definition of carried dependencies. The fcllowing for expression realizes a vector-vector product:

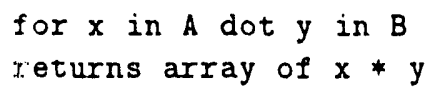


Table 2. The effectiveness of update-in-place and build-in-place analysis: Conc'rent-Vector.

\begin{tabular}{|c||c|c||c|c|c|}
\hline \multicolumn{6}{|c|}{ Alliant FX/80 } \\
\hline Program & NO OPT & OPT & Speedup & Updated-in-place & Built-in-place \\
\hline \hline GAUSSJ & 39.5 & 0.9 & $43 \mathrm{x}$ & $100 \%$ & $100 \%$ \\
\hline RICARD & 91.0 & 1.8 & $50 \mathrm{x}$ & $99 \%$ & $100 \%$ \\
\hline BMK11A & 1332.3 & 3.6 & $370 \mathrm{x}$ & $100 \%$ & $100 \%$ \\
\hline LOOPS & 8517.0 & 134.0 & $63 \mathrm{x}$ & $100 \%$ & $100 \%$ \\
\hline SIMPLE & 1790.9 & 32.9 & $54 \mathrm{x}$ & $99 \%$ & $99 \%$ \\
\hline WEATHER & 1195.3 & 13.6 & $87 \mathrm{x}$ & $100 \%$ & $93 \%$ \\
\hline
\end{tabular}

\section{end for}

By estimating execution costs, considering expression nesting levels, and applying knowledge of the target machine's instruction set, the compiler can easily chose the appropriate mode of parallel execution for these expressions: concurrent, vector, or concurrent-vector.

On the Alliant, we chose to compile Sisal to C, and use the resident $\mathrm{C}$ compiler to generate vector code. Because the SISAL compiler has a complete understanding of each program's data dependencies, it adds directives to the $\mathrm{C}$ code to help vectorization; that is, tells the $\mathrm{C}$ compiler exactly when it is safe to vectorize. This scheme simplifies code generation and improves portability. Further, it places the end responsibility for code quality on the resident compilers, where it should be.

\section{Current Results}

In this report we have praised applicative languages for their semantic aesthetics, described their potential inefficiencies, and highlighted some important optimizations. In this section we compare the performance of SISAL and ForTran using the benchmark suite in Table 1. The times (in seconds) were collected on an Alliant FX/80 using both 1 and 5 processors (ACEs). Before we present the numbers, let us summarize the features of each program and comment on their relevant execution parameters.

The first program is GAUSSJ. This benchmark solves a set of linear equations using Gauss-Jordan elimination with full pivoting; we ran a $100 \times 100$, double-precision problem. The second program, RICARD, is a production code developed at the University of Colorado Medical Center [6]. It simulates experimentally observed elution patterns of proteins and ligands in a column of gel. For the report, we ran a $15 \times 1215$ dimension problem (double precision) and timed the first 200 cycles $^{2}$ of execution $(40,000$ cycles make a complete production run). The third benchmark, BMK11A, is a particle in cell fragment developed at Los Alamos National Laboratory for analyzing Cray machine and compiler performance. All the computed arrays are one dimensional, each comprising 2560 double precision elements. We timed the first 10 cycles of execution. The fourth program, LOOPS, is the Livermore Loops [15]. We executed each of the loops 4000 times. SIMPLE ${ }^{3}$, a hydrodynamics benchmark developed at Lawrence Livermore National Laboratory, is the fifth program in the suite [8]. We ran a $100 \times 100$ double precision problem, and let it run to completion (which required 62 cycles). The last benchmark, and largest, is WEATHER [7]. This code, which was developed at Royal Melbourne Institute of Technology, is a one-level barotropic weather prediction code. The Department of Meteorology at the University of Melbourne provided the original FORTRAN version. Like RICARD, WEATHER is a production code. For this report, we timed the first 10 cycles of a $420 \mathrm{~km}$ problem. Unlike the other benchmarks, this one manipulates complex data.

In no case did we annotate the ForTran codes to help the compiler discover parallelism, or modify the underlying mathematical model of a particular problem without changing both the SISAL and ForTran versions. However, the SiSal codes wore written to exploit the data dependencies of the associated models, using for expressions wherever possible. Tables 3 and 4 give the performance data ${ }^{4}$. The former is with

\footnotetext{
${ }^{2}$ For all the benchunarks, a "cycle" refers to an iteraticn of the outermost loop.

${ }^{3}$ Different versions of SIMPLE exist. We used version 2 for this report.

${ }^{4}$ The compilers used on the Alliant $\mathrm{FX} / 80$ were Optimizing Sisal Compiler V6.5 with all optimizations enabled, $\mathrm{FX} / \mathrm{C}$ V2.1.23 (using - Ogiv - AS for vector operation and -Ogi - AS otherwise), and FX/Fortran V4.2.40 (using - Ogc-AS for concurrent operation and -OgCv -AS for concurrent-vector operation).
} 
Table 3. Performance data for the benchmarks: Concurrent.

\begin{tabular}{|c||c|c||c|c|}
\hline \multirow{2}{*}{\multicolumn{2}{c}{ Alliant FX/80 }} & \multicolumn{2}{c|}{ Concurrent } & \multicolumn{2}{c|}{ (IN SECONDS) } \\
\hline \multirow{2}{*}{ Program } & \multicolumn{2}{c|}{ One ACE } & \multicolumn{2}{c|}{ Five ACEs } \\
\cline { 2 - 5 } & Sisal & Fortran & Sisal & Fortran \\
\hline \hline GAUSSJ & 4.7 & 4.4 & 1.3 & 1.0 \\
\hline RICARD & $\mathbf{1 4 . 6}$ & 17.1 & $\mathbf{3 . 4}$ & 3.6 \\
\hline BMK11A & 6.8 & 15.2 & $\mathbf{3 . 2}$ & 4.5 \\
\hline LOOPS & $\mathbf{4 3 4 . 4}$ & 590.6 & $\mathbf{1 9 4 . 2}$ & 226.5 \\
\hline SIMPLE & $\mathbf{1 3 6 . 4}$ & 166.0 & $\mathbf{3 4 . 8}$ & 107.7 \\
\hline WEATHER & 63.0 & 60.6 & $\mathbf{1 5 . 0}$ & 25.4 \\
\hline
\end{tabular}

Table 4. Performance data for the benchmarks: Concurrent-Vector.

\begin{tabular}{|c||c|c||c|c|}
\hline \multirow{2}{*}{ Alliant FX/80 } & \multicolumn{3}{c|}{ Concurrent-Vector } & (IN SECONDS) \\
\hline \multirow{2}{*}{ Program } & \multicolumn{2}{c|}{ One ACE } & \multicolumn{2}{c|}{ Five ACEs } \\
\cline { 2 - 5 } & Sisal & Fortran & Sisal & Fortran \\
\hline \hline GAUSSJ & 2.6 & 1.7 & $\mathbf{0 . 9}$ & 1.0 \\
\hline RICARD & $\mathbf{7 . 3}$ & 7.7 & $\mathbf{1 . 8}$ & 1.8 \\
\hline BMK11A & $\mathbf{7 . 7}$ & 12.8 & $\mathbf{3 . 6}$ & 4.2 \\
\hline LOOPS & $\mathbf{2 8 9 . 7}$ & 296.8 & 134.0 & 122.3 \\
\hline SIMPLE & $\mathbf{1 2 8 . 7}$ & 128.8 & $\mathbf{3 2 . 9}$ & 99.6 \\
\hline WEATHER & $\mathbf{5 5 . 5}$ & 75.1 & $\mathbf{1 3 . 6}$ & 54.4 \\
\hline
\end{tabular}

Table 5. Performance data with memory management optimizations: Concurrent-Vector.

\begin{tabular}{|c|c|c|c|c|c|c|}
\hline \multirow[b]{3}{*}{ Program } & $\mathrm{FX} / 80$ & \multicolumn{3}{|c|}{ Concurrent-Vector } & \multirow{2}{*}{\multicolumn{2}{|c|}{$\frac{\text { IN SECONDS) }}{\text { Five ACEs }}$}} \\
\hline & \multicolumn{3}{|c|}{ One ACE } & Five ACEs & & \\
\hline & $\begin{array}{l}\text { Sisal } \\
\text { Before }\end{array}$ & \begin{tabular}{|l|} 
Sisal \\
After
\end{tabular} & Fortran & $\begin{array}{c}\text { Sisal } \\
\text { Before }\end{array}$ & $\begin{array}{l}\text { Sisal } \\
\text { After }\end{array}$ & Fortran \\
\hline GAUSSJ & 2.6 & 1.8 & 1.7 & 0.9 & 0.5 & 1.0 \\
\hline Loop21 & 18.1 & 9.8 & 7.0 & 6.2 & 2.2 & 2.5 \\
\hline
\end{tabular}


vectorization disabled. For the smaller codes, SISAL is clearly competitive; for the larger, it is superior. Automatic parallelization of the smaller ForTRAN codes was predictably successful, but the larger codes did not fare as well. Close examination of the results revealed that the lack of interprocedural analysis hurt concurrentization. For example, we hand analyzed -ach function and subroutine in SIMPLE and annotated the FORTRAN source accordingly. In essence, we identified the reentrant functions and subroutines. As a result, the five processor concurrent and concurrentvector execution times decreased to 42 and 35 seconds respectively. Now consider the dramatic performance degradation for the ForTran version of WEATHER when compiled for concurrent-vector operation. For contrast, we recompiled the program with concurrency disabled and vectorization enabled. Even this resulted in a 6 second increase in execution time compared to the one processor run in Table 3 . Suspecting that the vector lengths were too small to overcome setup costs, we recompiled the program for concurrentvector execution but requested run time tests to determine the best mode of operation (the -alt option). This resulted in a 5 processor execution time of 40 seconds, verifying our suspicion. It turns out that the SISAL compiler chose to vectorize 22 loops, while the FORTRAN compiler chose to vectorize 64 , of which $5 i$ executed concurrently. In general, the For'tran compiler spread the work too thin.

The SISAL codes did automatically reclaim memory during execution, and the respective overheads were reflected in the times. However, the compiler could have reduce the associated costs. For example, although we are eliminating most of the intermediate arrays and unnecessary copying in SISAL programs, we are not currently optimizing the code required to allocate and deallocate arrays and other large aggregates. Often these operations are loop invariant. To illustrate, we hand optimized the storage management operations in GAUSSJ and Loop 21 of LOOPS. Table 5 shows the results. Note the substantial improvements. We are currently automating this optimization and expect similar performance improvements for all the benchmarks.

\section{$6 \quad$ Future Directions}

In this report, we have further demonstrated that the applicative paradigm is a powerful tool for scientific computation. One can, without exception, reap the benefits of side effect free semantics and at the same time acyuire a performance edge. The results are better maintenance, increased portability, enlanced productivity, and (most importantly) guaranteed confidence and security. These qualities cannot be overlooked. Remember, tomorrow's world will be asynchronous, and in such a world, determinancy is priceless.

So we wonder, will ForTran 2000 don applicative and functional semantics? We believe it will. The imperative paradigm has served its purpose well, but it is now time for change.

\section{Acknowledgements}

We are grateful for the computer system resources provided by the Northeast Parallel Architectures Center at Syracuse University. We would also like to relay special thanks to Rod Oldehoeft, John Feo, Tom Deboni, and the rest of the SISAL community.

This work was supported by the Applied Mathematical Sciences subprogram of the Office of Energy Research, U.S. Department of Energy, by Lawrence Livermore National Laboratory under contract No. W7405-Eng-48.

\section{References}

[1] Arvind and R. Nikhil. Executing a program on the MIT tagged-token dataflow architecture. IEEE Transactions on Computers, 39(3):300-318, March 1990.

[2] Jeffrey M. Barth. A practical interprocedural data-flow analysis algorithm. Communucations of the $A C M, 21(9): 724-736$, September 1978.

[3] A. Bloss. Path Analysis and the Optmization of Non-strict functional Languages. PhD thesis, Yale University, Computer Science Department, New Haven, Connecticut, 1989. Avaiable as Research Report YALEU/DCS/RR-704.

[4] A. Bloss. Update analysis and the efficient implementation of functional aggregates. In The 4th International Conference on Functional Programming Languages and Computer Architecture, pages $26-38,1989$.

[5] D. C. Cann. Compilation Techniques for High Performance Applicative Computation. PhD ihesis, Colorado State University, Computer Science Department, Fort Collins, CO, 198\%.

[6] J. Cann, E. York, J. Stewart, J. Vera, and R. Maccion:. Small zone gel chromatography of interacting systems: Theoretical and experimental evaluation of elution profiles for kinetically controlled macromolecule-ligand reactions. Analytical Buochemistry, (175), December 1988.

[7] P. Chang and C. Egan. An implementation of a barotropic numerical weather prediction model in the functional language SISAL. In Proceedongs of 
the SIGPLAN 1990 Symposium on Principles and Practice of Parallel Programming, March 1990.

[8] W. P. Crowley, C. P. Henderson, and T. E. Kudy. The simple code. Technical Report. UCID 17715, Lawrence Livermore National Laboratory, Livermore, CA, Februrary 1978.

[9] K. Gopinath. Copy Elimination in Single Assignment Languages. PhD thesis, Computer Systems Laboratory, Stanford University, Stanford, CA, 1989.

[10] P. Huciak. Conception, evolution, and application of functional programming languages. $A C M C \mathrm{Com}$ puting Surveys, 21(3):359-411, September 1989.

[11] P. Hudak and A. Bloss. The aggregate update problem in functional programming systems. In Twelfth Annual ACM Conference of the Principles of Programming Languages, pages 300-313, January 1885 .

[12] J. Hughes. Why functional programming matters. The Computer Journal, 32(2):98-107, A pril 1989.

[13] B. J. MacLennan. Functional Programming Practice and Theory. Addison-Wesley Publishing Company, Reading, Massachusetts, 1990.

[14] J. R. McGraw, S. K. Skedzielewski, S. J. Allan, R. R. Oldehoeft, J. Glauert, C. Kirkham, W. Noyce, and R. Thomas. Sisal: Streams and iteration in a single assignment language: Reference manual version 1.2. Manual M-146, Rev. 1, Lawrence Livermore National Laboratory, Livermore, CA, March 1985.

[15] F. H. McMahon. The Livermore Fortran kernels: A computer test of the numerical performance range. Technical Report UCRL-53745, Lawrence Livermore National Laboratory, Livermore, CA, December 1986.

[16] J. E. Ranelletti. Graph Transformation Algorithms for Array Memory Optimization in Applicative Languages. $\mathrm{PhD}$ thesis, University of California at Davis, Computer Science Department, Davis, California, 1987.

[17] S. K. Skedzielewski and R. J. Simpson. A simple method to remove reference counting in applicative programs. In Proceedings of CONPAR 88, September 1988.

\section{Disclaimer}

This document was prepared as an account of work sponsored by an agency of the United States Government. Neither the United States Government nor the University of California nor any of their employees, makes any warranty, express or implied, or assumes any legal liability or responsibility for the accuracy, cornpleteness, or usefulness of any information, apparatus, product, or process disclosed, or represents that its use would not infringe privately owned rights. Reference herein to any specific commercial products, process, or service by trade name, trademark, manufacturer, or otherwise, does not necessarily constitute or imply its endorsement, recommendation, or favoring by the United States Government or the University of California. The views and opinions of authors expressed herein do not necessarily state or reflect those of the United States Government or the University of California, and shall not be used for advertising or product endorsement purposes. 

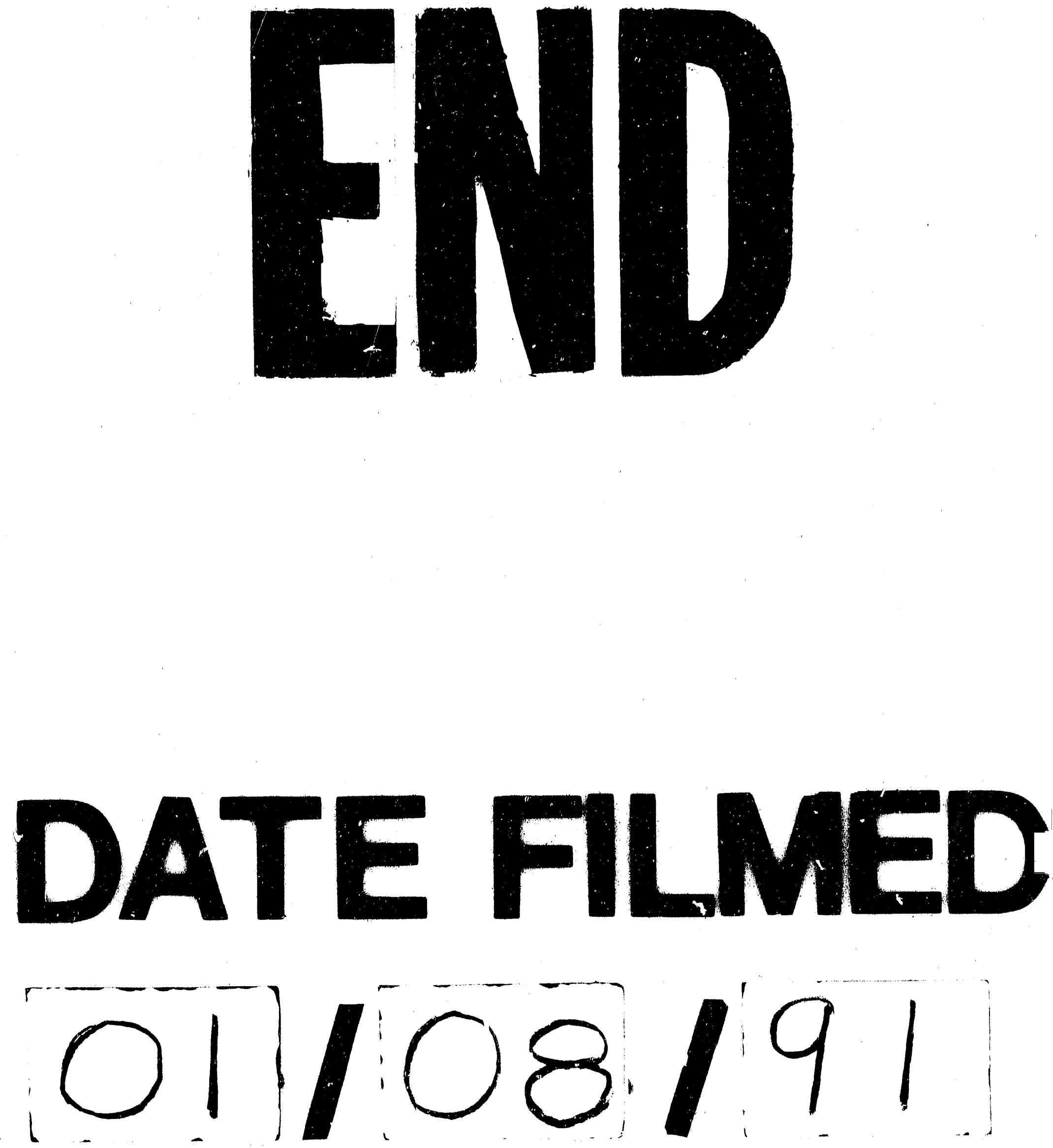
